\title{
A RARE CASE OF JEJUNAL DIVERTICULA PRESENTING AS PERFORATION: A CASE REPORT
}

Sushama Surapaneni ${ }^{1}$, Gollapalli Srinivas Veerraju², Vijaya Bhaskar Reddy A ${ }^{3}$

\section{HOW TO CITE THIS ARTICLE:}

Sushama Surapaneni, Gollapalli Srinivas Veerraju, Vijaya Bhaskar Reddy A. "A Rare case of Jejunal Diverticula presenting as Perforation: A Case Report". Journal of Evolution of Medical and Dental Sciences 2014; Vol. 3, Issue 17, April 28; Page: 4529-4532, DOI: 10.14260/jemds/2014/2470

ABSTRACT: Jejunal diverticula are a rare entity and usually asymptomatic. However, they may cause chronic non-specific symptoms or rarely lead to acute abdomen. The condition is difficult to diagnose because patients generally present with symptoms that mimic other diseases. It is important for clinicians to have awareness of this entity. The authors report a case of jejunal diverticulitis in a 75 year old male that was initially treated for other disease but eventually required specific surgical intervention.

KEYWORDS: Jejunal diverticula, perforation, acute abdomen.

INTRODUCTION: Jejunal diverticula are rare with an incidence of $<0.5 \% .^{1}$ One meaning of diverticulum is a wayside house of the ill-famed; certainly these wayside houses give rise to many pathological conditions. ${ }^{2}$ Diverticula can occur from the stomach to the rectosigmoid. They can be of congenital or acquired type. In the congenital diverticula all 3 coats of bowel are present while in acquired diverticula muscular layer is absent. Diverticula of small intestine are rare. They can be single or multiple. Diverticula in the jejunum tend to be large and multiple pathologically these are pseudo diverticula of pulsion type, resulted from increased intra-luminal pressure and weakening of the bowel wall. These outpouchings only contain mucosa and submucosa. Complications are reported in 10 to $30 \%$ of patients. These include chronic abdominal pain, malabsorption, hemorrhage, diverticulitis, obstruction, abscess formation and rarely diverticular perforation. They may cause chronic non-specific symptoms or rarely lead to acute presentation.

CASE REPORT: A 75 yr. old male patient presented to emergency department with obstructed left sided inguinal hernia. He is a known diabetic and hypertensive. Emergency surgery was done, contents of the sac were normal and pushed back in to the abdomen, sac was transfixed and hernioplasty was done. Surgery was uneventful and patient was stable for 2days. On $3^{\text {rd }}$ postoperative day patient developed abdominal distention and pain all over the abdomen. Guarding and rigidity was present physical examination revealed, Temperature $36^{\circ} \mathrm{C}$, Heart rate $105 / \mathrm{min}$, Blood pressure 130/50 mmHg, Respiratory rate 16 breaths/min. Abdominal examination revealed a generalized abdominal tenderness and signs of peritonitis. Rectum was empty and no mass was felt. Laboratory investigations revealed an elevated White cell count of $13500 \mathrm{cells} / \mathrm{mm}^{3}$ Renal profile was normal. Abdominal radiography shows multiple dilated loops of bowel. Patient was taken for emergency laparotomy which identified multiple perforated jejunal diverticulae. Figure (1) Primary resection of the diverticular segment and end to end anastomosis of the normal bowel done Figure (2). Extensive abdominal wash out was given and the patient recovered uneventfully. 


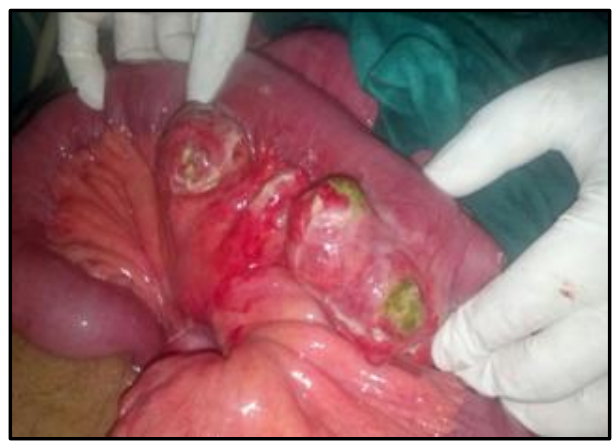

Fig. 1: (multiple jejunal divericulae with perforation)

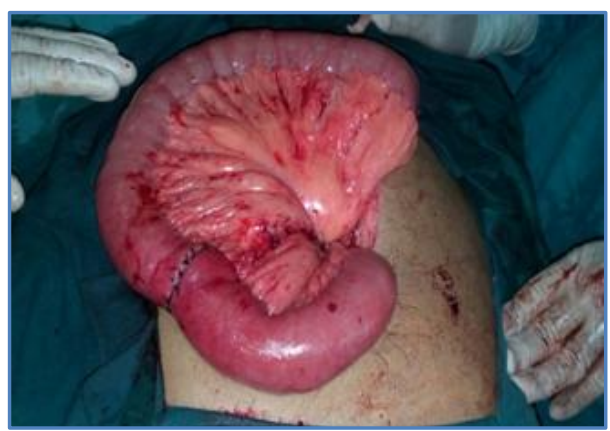

Fig. 2: (end to end anastomosis)

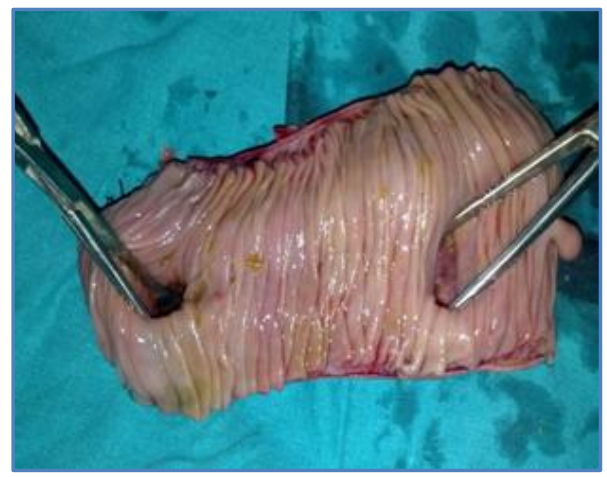

Fig. 3: (cut section of specimen)

DISCUSSION: Jejunal diverticula are pseudo diverticula which were first described by Somerling in 1794 and by Sir Astley Cooper in $1807 .^{3}$ Occurs mainly in elderly males around $6^{\text {th }}$ and $7^{\text {th }}$ decade..$^{4}$ The most common part of the small bowel to be affected by diverticula is the proximal jejunum (75\%), followed by the distal jejunum (20\%) and the ileum (5\%). ${ }^{4}$ These are false diverticula and multiple herniations of mucosa and sub mucosa through the weakest portion of bowel along the mesenteric side. These diverticula arise on the mesenteric border where the mesenteric vessels penetrate the jejunum. These develop as a result of abnormalities in peristalsis i.e., Intestinal dyskinesia and high segmental intra luminal pressure. The etiology is unclear. Krishnamurthy et al. ${ }^{5}$ focused on abnormalities of the smooth muscles or of the myenteric plexus in order to explain intestinal dyskinesia. Kongara et al. ${ }^{6}$ performed manometric studies of the small bowel and described functional abnormalities in patient with small bowel diverticula. Low fibre and fat rich diet intake is the most common cause. Progressive systemic sclerosis often involves the gastrointestinal tract and constitutes a characteristic example of proven dysmotility and acquired origin of the jejunoileal diverticulosis. Manometric studies, performed in patients with the disease, demonstrated intestinal dysmotility in $88 \%$ of the cases examined. ${ }^{7}$ Weston et al. ${ }^{8}$ reported an important incidence of small bowel dilation and diverticula (42\%) in patients with progressive systemic sclerosis.

Jejunal diverticula are clinically silent until they presents with complications associated with diverticular disease. They are usually found incidentally at laparotomy. Chronic symptoms are vague abdominal pain, localized either to the epigastrium or peri umbilical region, functional pseudo obstruction, anemia, fatigue, weakness, pedal edema. diarrhoea, flatulence and weight loss. Acute complications include intestinal obstruction, hemorrhage and perforation. 
Abdomen \& chest radiograph shows evidence of perforation, such as free air under the diaphragm or evidence of intestinal obstruction with multiple air-fluid levels and dilated loops small bowel. Endoscopic procedures like Double-balloon enteroscopy ${ }^{9}$ and capsule endoscopy are useful in diagnosing small-bowel disorders. Barium contrast of upper gastrointestinal tract useful in uncomplicated cases. CT with contrast shows thickening or inflammation of the jejunum or localized abscess formation. Diagnostic laparoscopy is useful in investigating patients with a complicated symptomatology, avoiding the need for unnecessary laparotomy. ${ }^{11}$

Tsiotos et al ${ }^{12}$ analyzed 112 cases of jejunoileal diverticulosis and of these, $42 \%$ of cases were Asymptomatic and require no treatment. The remaining patients had symptoms of diarrhoea (58\%), chronic abdominal pain (51\%) or bloating (44\%). Symptomatic cases often present with unspecific discomfort. That fact makes the clinical diagnosis difficult, as it was in the presented clinical case. Jejunal diverticulosis is a potential source of extensive bleedings. Diverticulitis with perforation and mechanical obstruction need surgical intervention. Segmental resection of the small bowel is the preferred method in these cases. ${ }^{13}$

\section{REFERENCES:}

1. Zager JS, Garbus JE, Shaw JP, Cohen MG, Garber SM. Jejunal diverticulosis: a rare entity with multiple presentations, a series of cases. Dig Surg: 17:643-645; 2000.

2. Ijaz Ahmed, Mohammad Naeem, Ambreen Samad, Amir Nasir, Zahid Aman. Complicated Jejunal Diverticula as Surgical Emergency; Ayub Med Coll Abbottabad; 22(1):2010.

3. Makris K, Tsiotos GG, Stafyla V, Sakorafas GH. Small intestinal nonmeckelian diverticulosis: clinical review. J Clin Gastroenterol.43:201-7; 2009.

4. Lempinen M, Salmela K, Kemppainen E. Jejunal diverticulosis: a potentially dangerous entity. Scand J Gastroenterol 2004, 39:905-9.

5. de Bree E, Grammatikakis J, Christodoulakis M, Tsiftsis D. The clinical significance of acquired jejunoileal diverticula. Am J Gastroenterol. 1998; 93:2523-8.

6. Krishnamurthy S, Kelly MM, Rohrmann CA, Schuffler MD. Jejunal diverticulosis. A heterogeneous disorder caused by a variety of abnormalities of smooth muscle or myenteric plexus. Gastrenterol 1983, 85:538-547.

7. Kongara KR, Soffer EE. Intestinal motility in small bowel diverticulosis: a case report and review of the literature. J Clin Gastroenterol 2000, 30:84-6.

8. Marie I, Levesque H, Ducrotté P, Denis P, Benichou J, Hellot MF et al. Courtois H. Manometry of the upper intestinal tract in patients with systemic sclerosis: a prospective study. Arthritis Rheum 1998, 41:1874-83.

9. Weston S, Thumshirn M, Wiste J, Camilleri M. Clinical and upper gastrointestinal motility features in systemic sclerosis and related disorders. Am J Gastroenterol 1998, 93:1085-9.

10. Yang CW, Chen YY, Yen HH, Soon MS. Successful double balloon enteroscopy treatment for bleeding jejunal diverticulum: A case report and review of the literature. J Laparoendos Adv Surg Techn 2009, 9:637-640.

11. Cross MJ, Snyder SK. Laparoscopic-directed small bowel resection for jejunal diverticulitis with perforation. J Laparoendosc Surg 1993, 3:47-49

12. Tsiotos GG, Farnell MB, Ilstup DM. Non-Meckelian jejunal or ileal diverticulosis: an analysis of 112 cases. Surgery 1994; 116: 726-32.

13. A. Surov, K. Stock Jejunal Diverticulosis. Eur J Med Res (2005) 10:358-360. 


\section{CASE REPORT}

\section{AUTHORS:}

1. Sushama Surapaneni

2. Gollapalli Srinivas Veerraju

3. Vijaya Bhaskar Reddy A.

\section{PARTICULARS OF CONTRIBUTORS:}

1. Associate Professor, Department of General Surgery, Konaseema Institute of Medical Sciences \& Research Foundation.

2. Assistant Professor, Department of General Surgery, Konaseema Institute of Medical Sciences \& Research Foundation.

3. Post Graduate, Department of General Surgery, Konaseema Institute of Medical Sciences \& Research Foundation.

\section{NAME ADDRESS EMAIL ID OF THE CORRESPONDING AUTHOR:}

Dr. Sushama Surapaneni, Associate Professor,

KIMS, Department of Surgery, Amalapuram, Andhra Pradesh. E-mail: sushama720@gmail.com

Date of Submission: 06/01/2014.

Date of Peer Review: 07/01/2014.

Date of Acceptance: 18/04/2014.

Date of Publishing: 24/04/2014. 